\title{
NON-REALIZABILITY OF THE PURE BRAID GROUP AS AREA-PRESERVING HOMEOMORPHISMS
}

\author{
LEI CHEN
}

\begin{abstract}
Let Homeo $+\left(D_{n}^{2}\right)$ be the group of orientation-preserving homeomorphisms of $D^{2}$ fixing the boundary pointwise and $n$ marked points as a set. Nielsen realization problem for the braid group asks whether the natural projection $p_{n}: \operatorname{Homeo}_{+}\left(D_{n}^{2}\right) \rightarrow B_{n}:=\pi_{0}\left(\operatorname{Homeo}_{+}\left(D_{n}^{2}\right)\right)$ has a section over subgroups of $B_{n}$. All of the previous methods either use torsions or Thurston stability, which do not apply to the pure braid group $P B_{n}$, the subgroup of $B_{n}$ that fixes $n$ marked points pointwise. In this paper, we show that the pure braid group has no realization inside the areapreserving homeomorphisms using rotation numbers.
\end{abstract}

\section{INTRODUCTION}

Denote by $D^{2}$ the 2-dimensional disk. Let $\operatorname{Homeo}_{+}\left(D_{n}^{2}\right)$ be the group of orientation-preserving homeomorphisms of $D^{2}$ fixing the boundary pointwise and $n$ marked points as a set. Denote by $B_{n}:=\pi_{0}\left(\operatorname{Homeo}_{+}\left(D_{n}^{2}\right)\right)$. The Nielsen realization problem for $B_{n}$ asks whether the natural projection

$$
p_{n}: \mathrm{Homeo}_{+}\left(D_{n}^{2}\right) \rightarrow B_{n}
$$

has a section over subgroups of $B_{n}$. For the whole group $B_{n}$, this question has several previous results. Salter-Tshishiku [ST16] uses Thurston stability to show that $B_{n}$ has no realization in $\operatorname{Diff}_{+}\left(D_{n}^{2}\right)$ and the author [Che19] uses "hidden torsions" and Markovic's machinery [Mar07] to show that $B_{n}$ has no realization in $\operatorname{Homeo}_{+}\left(D_{n}^{2}\right)$. Let $P B_{n}<B_{n}$ be the subgroup that preserves $n$ marked points pointwise. The Nielsen realization problem for $P B_{n}$ is widely open since the two methods in [ST16] and [Che19] fail to work and has no hope to repair. The following question is asked by [MT18, Question 3.12] and [ST16, Remark 1.4].

Problem 1.1 (Realization of pure braid group). Does $P B_{n}$ have realization as diffeomorphisms or homeomorphisms? In other words, does $p_{n}$ have sections over $P B_{n}$ ?

Denote by $\operatorname{Homeo}_{+}^{a}\left(D_{n}^{2}\right)$ the group of orientation-preserving, area-preserving homeomorphisms of $D^{2}$ fixing the boundary pointwise and $n$ marked points as a set. In this paper, we make a progress proving the following result.

Theorem 1.2. The pure braid group cannot be realized as area-preserving homeomorphisms on $D_{n}^{2}$ for $n \geq 9$. In other words, the natural projection $p_{n}^{a}$ : $\operatorname{Homeo}_{+}^{a}\left(D_{n}^{2}\right) \rightarrow B_{n}$ has no sections over $P B_{n}$.

We remark that the Nielsen realization problem is closely related to the existence of flat structures on a surface bundle. We refer the reader to [MT18] for more history and background. 
Comparing with the method in [CM19]. The novelty of this paper is to provide a different ending towards [CM19]. The original ending is to use the fact that certain Dehn twist is a product of commutator in its centralizer. However, such structure does not hold in $P B_{n}$. Instead, we prove a stronger dynamical property about Dehn twists about non-separating curves. In the beginning of Section 4, we present an outline of the proof. Since this paper has a lot of overlap with [CM19], we omit or sketch many proofs to reduce redundancy.

\section{Organization of the paper.}

- In Section 2, we discuss rotation numbers;

- In Section 3, we discuss the pure braid group and the minimal decomposition theory;

- In Section 4, we give an outline of the proof and finish the argument.

Acknowledgement. The author would like to thank Vlad Markovic for helpful discussion.

\section{ROTATION NUMBERS OF ANNULUS HOMEOMORPHISMS}

In this section, we discuss the properties of rotation numbers on annuli.

2.1. Rotation number of an area-preserving homeomorphism of an annulus. Firstly, we define the rotation number for geometric annuli. Let

$$
N=N(r)=\left\{w \in \mathbb{C}: \frac{1}{r}<|w|<r\right\}
$$

be the geometric annulus in the complex plane $\mathbb{C}$. Denote the geometric strip in $\mathbb{C}$ by

$$
P=P(r)=\left\{x+i y=z \in \mathbb{C}:|y|<\frac{\log r}{2 \pi}\right\} .
$$

The map $\pi(z)=e^{2 \pi i z}$ is a holomorphic covering map $\pi: P \rightarrow N$. The deck transformation on $P$ is $T(x, y)=(x+1, y)$.

Denote by $p_{1}: P \rightarrow \mathbb{R}$ the projection to the $x$-coordinate, and by $\mathrm{Homeo}_{+}(N)$ the group of homeomorphisms of $N$ that preserves orientation and the two ends. Fix $f \in \mathrm{Homeo}_{+}(N)$, and $x \in N$, and let $\widetilde{x} \in P$ and $\widetilde{f} \in \operatorname{Homeo}_{+}(P)$ denote lifts of $x$ and $f$ respectively. We define the translation number of the lift $\widetilde{f}$ at $\widetilde{x}$ by

$$
\rho(\widetilde{f}, \widetilde{x}, P)=\lim _{n \rightarrow \infty}\left(p_{1}\left(\widetilde{f}^{n}(\widetilde{x})\right)-p_{1}(\widetilde{x})\right) / n .
$$

The rotation number of $f$ at $x$ is then defined as

$$
\rho(f, x, N)=\rho(\widetilde{f}, \widetilde{x}, P) \quad(\bmod 1) .
$$

The rotation number is not defined everywhere (see, e.g., [Fra03] for more background on rotation numbers). The closed annulus $N_{c}$ is

$$
N_{c}=\left\{\omega \in \mathbb{C}: \frac{1}{r} \leq|\omega| \leq r\right\},
$$

For $f \in \mathrm{Homeo}_{+}\left(N_{c}\right)$, the rotation and translation numbers are defined analogously.

Let $A$ be an open annulus embedded in a Riemann surface (in particular this endows $A$ with the complex structure). By the Riemann mapping theorem, there is a unique $N(r)=N$ and a conformal 
map $u_{A}: A \rightarrow N$. For any $f \in \operatorname{Homeo}_{+}(A)$ (the group of end-preserving homeomorphisms), we define the rotation number of $f$ on $A$ by

$$
\rho(f, x, A):=\rho\left(g, u_{A}(x), N\right)
$$

where $g=u_{A} \circ f \circ u_{A}^{-1}$.

We have the following theorems of Poincaré-Birkhoff and Handel about rotation numbers [Han90] (See also Franks [Fra03]).

Theorem 2.1 (Properties of rotation numbers). If $f: N_{c} \rightarrow N_{c}$ is an orientation preserving, boundary component preserving, area-preserving homeomorphism and $\tilde{f}: P_{c} \rightarrow P_{c}$ is any lift, then:

- (Handel) The translation set

$$
R(\tilde{f})=\bigcup_{\widetilde{x} \in P_{c}} \rho\left(\tilde{f}, \widetilde{x}, P_{c}\right)
$$

is a closed interval.

- (Poincaré-Birkhoff) If $r \in R(\widetilde{f})$ is rational, then there exists a periodic orbit of $f$ realizing the rotation number $r$ mod 1 .

2.2. Separators and its properties. We let $A$ continue to denote an open annulus embedded in a Riemann surface. Then $A$ has two ends and we choose one of them to be the left end and the other one to be the right end. We call a subset $X \subset A$ separating (or essential) if every arc $\gamma \subset A$ which connects the two ends of $A$ must intersect $X$.

Definition 2.2 (Separator). We call a subset $M \subset A$ a separator if $M$ is compact, connected and separating.

The complement of $M$ in $A$ is a disjoint union of open sets. We have the following lemma.

Lemma 2.3. Let $M$ be a separator. Then there are exactly two connected components $A_{L}(M)$ and $A_{R}(M)$ of $A-M$ which are open annuli homotopic to $A$ and with the property that $A_{L}(M)$ contains the left end of $A$ and $A_{R}(M)$ contains the right end of $A$. All other components of $A-M$ are simply connected.

Proof. We compactify the annulus $A$ by adding points $p_{L}$ and $p_{R}$ to the corresponding ends of $A$. The compactifications is a two sphere $S^{2}$. Moreover, $M$ is a compact and connected subset of $S^{2}-\left\{p_{L}, p_{R}\right\}$.

Now, we observe that every component of $S^{2}-M$ is simply connected. Denote by $\Omega_{L}$ and $\Omega_{R}$ the connected components of $S^{2}-M$ containing $p_{L}$ and $p_{R}$ respectively. Since $M$ is separating we conclude that these are two different components. We define $A_{L}(M)=\Omega_{L}-p_{L}$ and $A_{R}(M)=$ $\Omega_{R}-p_{R}$. It is easy to verify that these are required annuli.

We now prove another property of a separator. Let $\pi: \widetilde{A} \rightarrow A$ be the universal cover.

Proposition 2.4. Let $M \subset A$ be a compact domain with smooth boundary. Then $\pi^{-1}(M)$ is connected. 
Proof. Since $M$ is a compact domain with boundary which separates the two ends of $A$, we can find a circle $\gamma \subset M$ which is essential in $A$ (i.e. $\gamma$ is a separator itself) (note that $M$ has only finitely many boundary components). Denote by $T$ the deck transformation of $\widetilde{A}$. Thus, the lift $\pi^{-1}(\gamma)$ is a $T$-invariant, connected subset of $\widetilde{A}$. Let $C$ be the component of $\pi^{-1}(M)$ which contains $\pi^{-1}(\gamma)$. Then $C$ is $T$ invariant. We show $\pi^{-1}(M)=C$.

Let $p \in M$. Since $M$ is a compact domain with smooth boundary, we can find an embedded closed arc $\alpha \subset M$ which connects $p$ and $\gamma$. Let $\widetilde{p}$ be a lift of $p$ and let $\widetilde{\alpha}$ be the corresponding lift of $\alpha$ such that $\widetilde{p}$ is one of its endpoints. Then, the other endpoint of $\widetilde{\alpha}$ is in $\pi^{-1}(\gamma)$, and this shows that $\tilde{p} \in C$. This concludes the proof.

Now we discuss an ordering on the set of separators.

Proposition 2.5. Suppose $M_{1}, M_{2} \subset A$ are two disjoint separators. Then either $M_{1} \subset A_{L}\left(M_{2}\right)$ or $M_{1} \subset A_{R}\left(M_{2}\right)$. Moreover, $M_{1} \subset A_{L}\left(M_{2}\right)$ implies $M_{2} \subset A_{R}\left(M_{1}\right)$.

Proof. Since $M_{1}$ is connected it follows that $M_{1}$ is a subset of a connected component $C$ of $A-M_{2}$. Since $C$ is open, we know that there is a neighborhood $N_{1}$ of $M_{1}$ with smooth boundary such that $N_{1} \subset C$ (It is elementary to construct such $N_{1}$ ). If $C$ is simply connected, the cover $\pi^{-1}(C) \rightarrow C$ is a trivial cover. Let $\widetilde{C}$ be a connected component of $\pi^{-1}(C)$. By Proposition 2.4 the set $\pi^{-1}\left(N_{1}\right)$ is connected so it is contained in a single connected component of $\pi^{-1}(C)$. However, this contradicts the fact that $\pi^{-1}\left(N_{1}\right)$ is also translation invariant. Thus, either $M_{1} \subset A_{L}\left(M_{2}\right)$ or $M_{1} \subset A_{R}\left(M_{2}\right)$.

Suppose $M_{1} \subset A_{L}\left(M_{2}\right)$. Then $A_{L}\left(M_{1}\right) \subset A_{L}\left(M_{2}\right)$ as well. On the other hand, by the first part of the proposition we already know that either $M_{2} \subset A_{L}\left(M_{1}\right)$ or $M_{2} \subset A_{R}\left(M_{1}\right)$. If $M_{2} \subset A_{L}\left(M_{1}\right)$, then $A_{L}\left(M_{2}\right) \subset A_{L}\left(M_{1}\right)$. This shows that $A_{L}\left(M_{1}\right) \subset A_{L}\left(M_{2}\right)$ which implies that $M_{2} \subset A_{L}\left(M_{2}\right)$. This is absurd so we must have $M_{2} \subset A_{R}\left(M_{1}\right)$.

Definition 2.6. The inclusion $M_{1} \subset A_{L}\left(M_{2}\right)$ is denoted as $M_{1}<M_{2}$.

2.3. The rotation interval of an annular continuum and prime ends. Let $K \subset A$ be a separator (in literature also known as an essential continuum). We call $K$ an essential annular continuum if $A-K$ has exactly two components. Observe that an essential annular continuum can be expressed as a decreasing intersection of essential closed topological annuli in $A$.

It is possible to turn any separator $M \subset A$ into an essential annular continuum. Let $M$ be a separating connected set. By Lemma 2.3, we know that $A-M$ has exactly two connected annular components $A_{L}(M)$ and $A_{R}(M)$, and all other components of $A-M$ are simply connected. We call a simply connected component of $A-M$ a bubble component. Then the annular completion $K(M)$ of $M$ is defined as the union of $M$ and the corresponding bubble components of $A-M$.

Proposition 2.7. Let $M \subset A$ be a separator. Then the annular completion $K(M)$ is an annular continuum.

Proof. We can again compactify $A$ by adding the points $p_{L}$ and $p_{R}$, one at each end. The compactification is the two sphere $S^{2}$. Then $A_{L}(M)$ and $A_{R}(M)$ are two disjoint open discs in $S^{2}$, 
and $K(M)=S^{2}-\left(A_{L}(M) \cup A_{R}(M)\right)$. But the complement of two disjoint open discs in $S^{2}$ is connected. This proves the proposition.

Now let $f$ be a homeomorphism of $A$ that leaves an annular continuum $K$ invariant. If $\mu$ is an invariant Borel probability measure supported on $K$, we define the $\mu$-rotation number

$$
\sigma(f, \mu)=\int_{A} \phi d \mu
$$

where $\phi: A \rightarrow \mathbb{R}$ is the function which lifts to the function $p_{1} \circ f-p_{1}$ on $\widetilde{A}$ (recall that $p_{1}: \widetilde{A} \rightarrow \mathbb{R}$ is the projection onto the first coordinate).

The set of $f$ invariant Borel probability measures on $K$ is a non empty, convex, and compact set (with respect to the weak topology on the space of measures). We define the rotation interval of $K$

$$
\sigma(f, K)=\{\sigma(f, \mu) \mid \mu \in M(K)\}
$$

which is a non-empty segment $[\alpha, \beta]$ of $\mathbb{R}$. The interval is non empty because there exists at least one $f$ invariant measure, and it is an interval because the set of $f$ invariant measures is convex.

The following is a classical result of Franks-Le Calvez [FC03, Corollary 3.1].

Proposition 2.8. If $\sigma(f, K)=\{\alpha\}$, the sequence

$$
\frac{p_{1} \circ f^{n}(x)-p_{1}(x)}{n}
$$

converges uniformly for $x \in \pi^{-1}(K)$ to the constant function $\alpha$. This implies that points in $K$ all have the rotation number $\alpha$.

The following theorem of Franks-Le Calvez [FC03, Proposition 5.4] is a generalization of the Poincaré-Birkhoff Theorem.

Theorem 2.9. If $f$ is area-preserving and $K$ is an annular continuum, then every rational number in $\sigma(f, K)$ is realized by a periodic point in $K$.

The theory of prime ends is an important tool in the study of 2-dimensional dynamics which can be used to transform a 2-dimensional problem into a 1-dimensional problem. Recall that we assume that $A$ is an open annulus embedded in a Riemann surface $S$. Suppose that $f$ is a homeomorphism of $S$ which leaves $A$ invariant. Furthermore, let $K \subset A$ be an annular continuum and suppose that $f$ leaves $K$ invariant. Then both $A_{L}(K)$ and $A_{R}(K)$ are $f$ invariant.

Since $A$ is embedded in $S$, we can define the frontiers of $A, A_{L}(K)$, and $A_{R}(K)$. By Carathéodory's theory of prime ends (see, e.g., [Mil06, Chapter 15]), the homeomorphism $f$ yields an action on the frontiers of $A_{L}(K)$ and $A_{R}(K)$. Consider the right hand frontier of $A_{L}(K)$ (the one which is contained in $A$ ). Then the set of prime ends on this frontier is homeomorphic to the circle, and we denote by $f_{L}$ the induced homeomorphism of this circle. Likewise, the set of prime ends on left hand frontier of $A_{R}(K)$ is homeomorphic to the circle, and we denote by $f_{R}$ the induced homeomorphism this circle. 
The rotation number of a circle homeomorphism (defined by Equation (2)), is well defined everywhere and is the same number for any point on the circle. The rotation numbers of $f_{L}$ and $f_{R}$ are called $r_{L}$ and $r_{R}$. We refer to them as the left and right prime end rotation numbers of $f$. We have the following theorem of Matsumoto [Mat12.

Theorem 2.10 (Matsumoto's theorem). If $K$ is an annular continuum, then its left and right prime ends rotation numbers $r_{L}, r_{R}$ belong to the rotation interval $\sigma(f, K)$.

\section{Minimal DeCompositions And CHARACteristic ANNUli}

3.1. Minimal decompositions. We recall the theory of minimal decompositions of surface homeomorphisms. This is established in [Mar07]. Firstly we recall the upper semi-continuous decomposition of a surface; see also Markovic [Mar07, Definition 2.1]. Let $M$ be a surface.

Definition 3.1 (Upper semi-continuous decomposition). Let $\mathbf{S}$ be a collection of closed, compact, connected subsets of $M$. We say that $\mathbf{S}$ is an upper semi-continuous decomposition of $M$ if the following holds:

- If $S_{1}, S_{2} \in \mathbf{S}$, then $S_{1} \cap S_{2}=\emptyset$.

- If $S \in \mathbf{S}$, then $E$ does not separate $M$; i.e., $M-S$ is connected.

- We have $M=\bigcup_{S \in \mathbf{S}} S$.

- If $S_{n} \in \mathbf{S}, n \in \mathbb{N}$ is a sequence that has the Hausdorff limit equal to $S_{0}$ then there exists $S \in \mathbf{S}$ such that $S_{0} \subset S$.

Now we define acyclic sets on a surface.

Definition 3.2 (Acyclic sets). Let $S \subset M$ be a closed, connected subset of $M$ which does not separate $M$. We say that $S$ is acyclic if there is a simply connected open set $U \subset M$ such that $S \subset U$ and $U-S$ is homeomorphic to an annulus.

The simplest examples of acyclic sets are a point, an embedded closed arc and an embedded closed disk in $M$. Let $S \subset M$ be a closed, connected set that does not separate M. Then $S$ is acyclic if and only if there is a lift of $S$ to the universal cover $\widetilde{M}$ of $M$, which is a compact subset of $\widetilde{M}$. The following theorem is a classical result called Moore's theorem; see, e.g., Mar07, Theorem 2.1].

Theorem 3.3 (Moore's theorem). Let $M$ be a surface and $\mathbf{S}$ be an upper semi-continuous decomposition of $M$ so that every element of $\mathbf{S}$ is acyclic. Then there is a continuous map $\phi: M \rightarrow M$ that is homotopic to the identity map on $M$ and such that for every $p \in M$, we have $\phi^{-1}(p) \in \mathbf{S}$. Moreover $\mathbf{S}=\left\{\phi^{-1}(p) \mid p \in M\right\}$.

We call the map $M \rightarrow M / \sim$ the Moore map where $x \sim y$ if and only if $x, y \in S$ for some $S \in \mathbf{S}$. The following definition is [Mar07, Definition 3.1]

Definition 3.4 (Admissible decomposition). Let $\mathbf{S}$ be an upper semi-continuous decomposition of $M$. Let $G$ be a subgroup of $\operatorname{Homeo}(M)$. We say that $\mathbf{S}$ is admissible for the group $G$ if the following holds: 
- Each $f \in G$ preserves setwise every element of $\mathbf{S}$.

- Let $S \in \mathbf{S}$. Then every point, in every frontier component of the surface $M-S$ is a limit of points from $M-S$ which belong to acyclic elements of $\mathbf{S}$.

If $G$ is a cyclic group generated by a homeomorphism $f: M \rightarrow M$ we say that $\mathbf{S}$ is an admissible decomposition of $f$.

An admissible decomposition for $G<\operatorname{Homeo}(M)$ is called minimal if it is contained in every admissible decomposition for $G$. We have the following theorem [Mar07, Theorem 3.1].

Theorem 3.5 (Existence of minimal decompositions). Every group $G<\operatorname{Homeo}(M)$ has a unique minimal decomposition.

Denote by $\mathbf{A}(G)$ the sub collection of acyclic sets from $\mathbf{S}(G)$. By a mild abuse of notation, we occasionally refer to $\mathbf{A}(G)$ as a subset of $S_{g}$ (the union of all sets from $\mathbf{A}(G)$ ). To distinguish the two notions we do the following. When we refer to $\mathbf{A}(G)$ as a collection then we consider it as the collection of acyclic sets. When we refer to as a set (or a subsurface of $S_{g}$ ) we have in mind the other meaning.

We have the following result [Mar07, Proposition 2.1].

Proposition 3.6. Every connected component of $\mathbf{A}(G)$ (as a subset of $S_{g}$ ) is a subsurface of $M$ with finitely many ends.

Lemma 3.7. For $H<G<\operatorname{Homeo}(M)$, we have that $\mathbf{A}(G) \subset \mathbf{A}(H)$.

Proof. The inclusion $\mathbf{A}(G) \subset \mathbf{A}(H)$ is because that the minimal decomposition of $G$ is also an admissible decomposition of $H$ and the minimal decomposition of $H$ is finer than that of $G$.

3.2. Lifting through hyper-ellpitic branched cover. Denote by $S_{g ; n, b}$ the surface of genus $g$ with $b$ boundary components and $n$ marked points. To make the analysis easier, we take the following hyper-elliptic $\mathbb{Z} / 2$ branched covers

$$
\pi_{n}: S=S_{\frac{n-1}{2} ; n, 1} \rightarrow S_{0 ; n, 1} \text { for } n \text { odd or } \pi_{n}: S=S_{\frac{n}{2}-1 ; n, 2} \rightarrow S_{0 ; n} \text { for } n \text { even. }
$$

The cover is shown by the following figures. The hyperelliptic involution on $S$ is denoted by $\tau$.

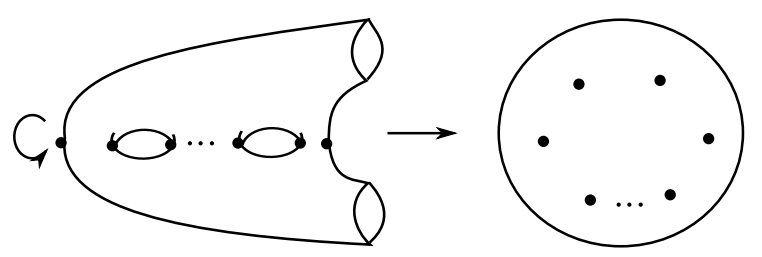

FIGURE 1. $n$ even

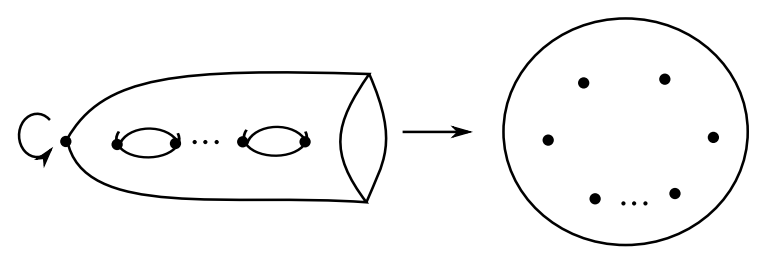

Figure 2. $n$ odd

Denote by $\widetilde{P B}_{n}$ the lifts of mapping classes under $\pi_{n}$, where it satisfies the following

$$
1 \rightarrow \mathbb{Z} / 2 \rightarrow \widetilde{P B}_{n} \stackrel{L}{\rightarrow} P B_{n} \rightarrow 1 \text {. }
$$

Let $c$ be a simple closed curve on $S_{0 ; n, 1}$ and denote by $T_{c}$ the Dehn twist about $c$. For every simple closed curve $c$ on $S_{0 ; n, 1}$, we have the following easy fact about its preimage under $\pi_{n}$. 
Fact 3.8. (1) If $c$ bounds odd number of points, then the lift is a single curve $c^{\prime}$. The preimage of $T_{c}^{2}$ under $L$ are $T_{c^{\prime}}$ and $T_{c^{\prime}} \tau$.

(2) If $c$ bounds even number of points, then the lift is two curves $c_{1}, c_{2}$. The preimage of $T_{c}$ under $L$ are $T_{c_{1}} T_{c_{2}}$ and $T_{c_{1}} T_{c_{2}} \tau$. In particular, if $c$ bounds 2 points, then $c_{1}=c_{2}$.

From the above fact, we know that if $c$ bounds 2 points and $c_{1}=c_{2}$ are the lifts, we have that $T_{c_{1}}^{2} \in \widetilde{P B}_{n}$. We have the following.

Fact 3.9. If $\alpha$ is a nonseparating simple closed curve that is invariant under $\tau$, then a square of the Dehn twist about $c$ is in $\widetilde{P B}_{n}$. We call such element an invariant Dehn twist square.

Let $b$ be the curve in $D_{n}^{2}$ bounding 5 points $P_{1}, \ldots, P_{5}$. The lift of $b$ under the cover $\pi_{n}$ is a curve $c$ bounding a genus 2 subsurface as the following figure.

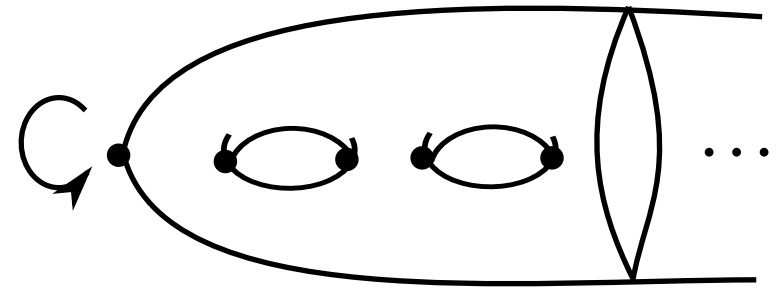

Figure 3. the curve $c$ bounding a genus 2 surface is the lift of a curve bounding 5 points

If a curve $\alpha$ is on the genus 2 subsurface of $S$ that is cut out by $c$, then we call the invariant Dehn twist square about $\alpha$ a left invariant Dehn twist square. We have the following important relations in $\widetilde{P B}_{n}$.

Proposition 3.10. The element $T_{c} \in \widetilde{P B}_{n}$ is a product of left invariant Dehn twist squares in $\widetilde{P B}_{n}$.

Proof. We have the basic fact that $T_{b}$ is generated by Dehn twists about curves in the interior of $b$ bounding 2 points; see, e.g., [FM12, Chapter 9]. Take a lift of all of the elements, we obtain that a product of squares of Dehn twists about nonseparating curves that are disjoint from $c$ and on the left of $c$ in $\widetilde{P B}_{n}$. After taking the square of the equation, we obtain the proposition.

3.3. Characteristic annuli. From now on, we work with the assumption that there exists a realization of the pure braid group

$$
\mathcal{E}^{\prime}: P B_{n} \rightarrow \operatorname{Homeo}_{+}^{a}\left(D_{n}^{2}\right) .
$$

Lifting by the hyperelliptic involution, we obtain a new realization

$$
\mathcal{E}: \widetilde{P B}_{n} \rightarrow \operatorname{Homeo}_{+}^{a}\left(S_{g}\right)^{\tau}
$$

where the image lies in the centralizer of the hyper-elliptic involution $\tau$. We now only work with the new realization $\mathcal{E}$. 
For an element $f \in \widetilde{P B}_{n}$, or a subgroup $F<\widetilde{P B}_{n}$, we shorten $\mathbf{A}(\varepsilon(f))$ as $\mathbf{A}(f)$, and $\mathbf{A}(\varepsilon(F))$ as $\mathbf{A}(F)$, to denote the corresponding collections of acyclic components. Denote by $S$ the hyperelliptic cover we defined in Section 3.2. Recall that $c \subset S$ is a separating curve that is invariant under $\tau$ that divides $S$ into subsurfaces $S_{L}$ of genus 2 and $S_{R}=S-S_{L}$ (see more about $c$ in the previous section). We know that $T_{c} \in \widetilde{P B}_{n}$. We have the following theorem about the minimal decomposition of $\mathcal{E}\left(T_{c}\right)$.

Theorem 3.11. The set $\mathbf{A}\left(T_{c}\right)$ has a component $\mathbf{L}(c)$ which is homotopic to $S_{L}$ and a component $\mathbf{R}(c)$ homotopic to $S_{R}$.

Proof sketch. The proof is the same as the proof of [CM19, Theorem 4.1]. We use the fact that there are pseudo-Anosov elements on the left and on the right of $c$ in $\widetilde{P B}_{n}$. In this theorem, we need $n \geq 9$.

For the rest of paper, denote by

$$
\mathbf{B}:=S-\mathbf{L}(c)-\mathbf{R}(c)
$$

Let $p_{L}: \mathbf{L}(c) \rightarrow \mathbf{L}(c) / \sim$ and $p_{R}: \mathbf{R}(c) \rightarrow \mathbf{R}(c) / \sim$ be the Moore maps of $\mathbf{L}(c)$ and $\mathbf{R}(c)$ corresponding to the decomposition $\mathbf{S}(c)$. Let $\mathbf{L} \subset \mathbf{L}(c) / \sim$ be an open annulus bounded by the end of $\mathbf{L}(c)^{\prime}$ on one side, and by a simple closed curve on the other. The open annulus $\mathbf{R} \subset \mathbf{R}(c) / \sim$ is defined similarly. We have the following definition (see [Mar07, Chapter 5]).

Definition 3.12. An annulus of the form $A=p_{L}^{-1}(\mathbf{L}) \cup \mathbf{B} \cup p_{R}^{-1}(\mathbf{R})$ is called a characteristic annulus.

Denote by $f=\mathcal{E}\left(T_{c}\right)$. Every characteristic annulus is invariant under $f$. We observe that $\mathbf{B}$ is a separator in $A$, that is, $\mathbf{B}$ is an essential, compact, and connected subset of $A$. Note that a characteristic annulus $A$ is invariant under $f$, but it may not be invariant under homeomorphisms which are lifts (with respect to $\mathcal{E}$ ) of other elements from $\widetilde{P B}_{n}$. However, $\mathbf{B}$ is invariant under these lifts of elements from the image under $\mathcal{E}$ of the centralizer of $T_{c}$ in $\widetilde{P B}_{n}$. As we see from the next lemma, the dynamical information about $f$ is contained in $\mathbf{B}$.

Lemma 3.13. Fix a characteristic annulus A. Then

(1) every number $0<r<1$ appears as the rotation number $\rho(f, x, A)$, for some $x \in A$,

(2) if $0<\rho(f, x, A)<1$, then $x \in B$.

The proof of the above lemma can be seen in [CM19, Lemma 4.5]. The reason is that $f$ is homotopic to a Dehn twist and that the realization is area-preserving.

\section{The Proof of Theorem 1.2}

In this section, we discuss the proof of Theorem 1.2. We now discuss the main strategy. 
4.1. Outline of the proof. Recall that $c$ is a separating simple closed curve that divides the surface $S$ (the hyper-elliptic cover of $S_{0 ; 1, n}$ ) into a genus 2 subsurface and the rest. Fix a characteristic annulus $A$. Let $E_{r}$ be the set of points in $A$ that have rotation numbers equal to $r$ under $\mathcal{E}\left(T_{c}\right)$. Lemma 3.13 states that the set $E_{r}$ is not empty when $0<r<1$.

The key observation of the proof lies in the analysis of connected components of $E_{r}$. Let $E$ be a component of $E_{r}$. We show the following:

(1) $E$ is $\mathcal{E}(h)$-invariant for $h$ a left invariant Dehn twist square,

(2) $\bar{E}$ is a separator in $A$,

(3) if $E$ contains a periodic orbit, then $E$ contains a separator.

Denote by $K(\bar{E})$ the annular completion of $\bar{E}$, and let $\rho\left(\mathcal{E}\left(T_{c}\right), K(\bar{E})\right)$ be the rotation interval of $K(\bar{E})$. We claim that $\rho\left(\mathcal{E}\left(T_{c}\right), K(\bar{E})\right)=\{r\}$. First of all, we know that $r \in \rho\left(\mathcal{E}\left(T_{c}\right), K(\bar{E})\right)$. If $\rho\left(\mathcal{E}\left(T_{c}\right), K(\bar{E})\right) \neq\{r\}$, then $\rho\left(\mathcal{E}\left(T_{c}\right), K(\bar{E})\right)$ contains infinitely many rational numbers. By Theorem 2.9 there exist three periodic points $x_{1}, x_{2}, x_{3} \in K(\bar{E})$ with different rational rotation numbers $r_{1}, r_{2}, r_{3}$. Let $F_{i}$ denote the connected component of $E_{r_{i}}$ containing $r_{i}$, and let $M_{i} \subset F_{i}$ be a separator.

By Proposition 2.5, there is an ordering on disjoint separators. Without loss of generality, we assume that $M_{1}<M_{2}<M_{3}$. Based on a discussion about the position $E$ with respect to $M_{i}$ 's, we obtain a contradiction. Thus, $\rho\left(\mathcal{E}\left(T_{c}\right), K(E)\right)$ is the singleton $\{r\}$.

We know from Theorem 2.10 that the left and right prime ends rotation numbers of $K(\bar{E})$ are both $r$. But in the group of circle homeomorphisms, the centralizer of an irrational rotation is essentially $S O(2)$.

We then show a new ingredient of the proof: the rotation numbers of the realization of a left invariant Dehn twist square on the set of prime ends of $K(\bar{E})$ are all 0 . This contradicts the fact that $T_{c}$ is a product left invariant Dehn twist squares as in Proposition 3.10 .

4.2. The set $E_{r}$. Once again we use abbreviation $f=\mathcal{E}\left(T_{c}\right)$. For a characteristic annulus $A$, we let

$$
E_{r}=\{x \in A: \rho(f, x, A)=r\} .
$$

By Lemma 3.13 , if $0<r<1$, we know that $E_{r}$ is nonempty and $E_{r} \subset \mathbf{B}$.

Next, we have the following key lemmas which corresponds to [CM19, Lemma 5.1, 5.3, 5.4].

Lemma 4.1. Fix $0<r<1$, and let $E$ denote a connected component of $E_{r}$. Fix a left invariant Dehn twist square $h$ in $\widetilde{P B}_{n}$. For $x \in E$, let $C(x) \in \mathbf{A}(h)$ be the corresponding acyclic set. Then $C(x) \subset E$. In particular, $E$ is $\mathcal{E}\left(C\left(T_{c}\right)\right)$-invariant.

Lemma 4.2. The closed set $\bar{E}$ is a separator (as defined in Section 2).

Lemma 4.3. Let $x$ be a periodic orbit of $f$ such that $\rho(f, x, A)=p / q$ and $0<p / q<1$. Then, the connected component $E$ of $E_{p / q}$ which contains $x$, also contains a separator (as a subset).

Fix an irrational number $r \in(0,1)$. By Lemma 3.13, we know that $E_{r}$ is not empty. Let $E$ be a connected component of $E_{r}$. By Lemma $\frac{4.1}{10}$, we know that $E$ is invariant under $\mathcal{E}\left(C\left(T_{c}\right)\right)$. 
By Lemma 4.2 , we know that $\bar{E}$ is a separator. The annular completions $K(\bar{E})$ of $\bar{E}$ is also $\mathcal{E}\left(C\left(T_{c}\right)\right.$ )-invariant since the definition is canonical. The following claim is at the heart of the entire construction.

Claim 4.4. Let $r_{L}$ and $r_{R}$ be the left and right prime ends rotation numbers of $f$ on $K(\bar{E})$. Then $r_{L}=r_{R}=r$.

Remark. We refer the reader to [CM19, Claim 5.2] for the proof. The only property we use about $\widetilde{P B}_{n}$ is Proposition 3.10 .

4.3. Finishing the proof. We need to show a new property of a left invariant Dehn twist square $h \in \widetilde{P B}_{n}$.

Theorem 4.5. The action of $\mathcal{E}\left(T_{b}^{2}\right)$ on the set of prime ends of $K(\bar{E})$ has rotation number 0.

Proof. Now we consider the rotation set of $\mathcal{E}\left(T_{b}^{2}\right)$ on $K(\bar{E})$. We claim that the rotation set satisfies

$$
\sigma\left(\mathcal{E}\left(T_{b}\right), K(\bar{E})\right)=\{0\}
$$

The reason is that if not, then it is a nontrivial closed interval. By Theorem 2.9, rational rotation numbers are realized by periodic orbit. However $K(\bar{E}) \subset B$, that means every point for $x \in$ $K(\bar{E}) \subset B$, there exists $C(x) \in \mathbf{A}\left(T_{b}^{2}\right)$ such that $C(x) \subset B$ by Lemma 4.1. However $C(x)$ is acyclic and fixed by $\mathcal{E}\left(T_{b}^{2}\right)$. Therefore, we know that the rotation number of $\mathcal{E}\left(T_{b}^{2}\right)$ on points in $C(x)$ is zero, which is a contradiction. Then by Theorem 2.10, we know that the rotation number of the action of $\mathcal{E}\left(T_{b}^{2}\right)$ on the set of prime ends is also zero.

We now finish the proof.

Proof. Since the rotation number of $\mathcal{E}\left(T_{c}\right)$ on the prime ends of $K(\bar{E})$ is an irrational number $r$, then it is semiconjugate to an irrational rotation. Then up to the same semiconjugation, the image of the centralizer of $T_{c}$ under $\mathcal{E}$ is $S O(2)$. The image of each element is determined by its rotation number. However, $\mathcal{E}\left(T_{c}\right)$ is a product of $\mathcal{E}\left(T_{b}^{2}\right)$ for $b$ nonseparating and invariant under $\tau$ by Proposition 3.10. By Lemma 4.5, we know that the rotation number of $\mathcal{E}\left(T_{b}^{2}\right)$ is zero. Thus their product should also have 0 rotation number. This contradicts the fact that the rotation number of $\mathcal{E}\left(T_{c}\right)$ is $r$, which is nonzero.

\section{REFERENCES}

[Che19] L. Chen. On the nonrealizability of braid groups by homeomorphisms. Geom. Topol., 23(7):3735-3749, 2019.

[CM19] L. Chen and V. Markovic. Non-realizability of the torelli group as area-preserving homeomorphisms. Preprint: https://arxiv.org/abs/1904.09490, 2019.

[FC03] J. Franks and PL. Calvez. Regions of instability for non-twist maps. Ergodic Theory Dynam. Systems, 23(1):111-141, 2003.

[FM12] B. Farb and D. Margalit. A primer on mapping class groups, volume 49 of Princeton Mathematical Series. Princeton University Press, Princeton, NJ, 2012. 
[Fra03] J. Franks. Rotation numbers and instability sets. Bull. Amer. Math. Soc. (N.S.), 40(3):263-279, 2003.

[Han90] M. Handel. The rotation set of a homeomorphism of the annulus is closed. Comm. Math. Phys., 127(2):339-349, 1990.

[Mar07] V. Markovic. Realization of the mapping class group by homeomorphisms. Invent. Math., 168(3):523-566, 2007.

[Mat12] S. Matsumoto. Prime end rotation numbers of invariant separating continua of annular homeomorphisms. Proc. Amer. Math. Soc., 140(3):839-845, 2012.

[Mil06] J. Milnor. Dynamics in one complex variable, volume 160 of Annals of Mathematics Studies. Princeton University Press, Princeton, NJ, third edition, 2006.

[MT18] K. Mann and B. Tshishiku. Realization problems for diffeomorphism groups. Preprint: https://arxiv.org/abs/1802.00490, 2018.

[ST16] N. Salter and B. Tshishiku. On the non-realizability of braid groups by diffeomorphisms. Bull. Lond. Math. Soc., 48(3):457-471, 2016.

Department of Mathematics

California Institute of Technology

Pasadena, CA 91125, USA

E-mail address: chenlei@caltech.edu 